\title{
Deconvolution and Causality for 1:1 Arrhythmia Discrimination in Dual Chamber Defibrillator
}

\author{
JL Rojo-Álvarez ${ }^{1}$, F Atienza ${ }^{2}$, N Martínez-Alzamora $^{3}$, A Arenal-Maíz ${ }^{4}$, \\ J Piñeiro-Ave ${ }^{1}$, A Artés-Rodríguez ${ }^{5}$ \\ ${ }^{1}$ DTSC, Universidad de Alcalá, Madrid, Spain \\ ${ }^{2}$ Hospital General Universitario, Valencia, Spain \\ ${ }^{3}$ Universidad Politécnica, Valencia, Spain \\ ${ }^{4}$ Hospital GU Gregorio Marañón, Madrid, Spain \\ ${ }^{5}$ DTSC, Universidad Carlos III de Madrid, Spain
}

\begin{abstract}
Automatic recognition of $1: 1$ ventricular tachycardia (VT) from supraventricular tachycardia (SVT) in dual chamber implantable cardioverter defibrillator $(I C D)$ is still an open issue. A new criterion is proposed, based on the modelling of a causal versus an anti-causal relationship between the atrial and the ventricular ICD stored electrograms (EGM). A cardiac activation model is presented, consisting on (a) a pulsed activation in the atria, (b) two impulse responses modelling the atrial and the ventricular depolarization, and (c) a filter describing the atrio-ventricular (AV) propagation delay. A mininum absolute error algorithm is developed, basing on template generation, deconvolution, stochastic gradient descent and projection onto convex sets. Examples of the algorithm when fitting the model to sinus rhythm (SR), SVT and VT records are employed. The algorithm is shown to be robust in the delay-filter order choice. We propose this method as a promising framework to design low computational burden 1:I SVT-VT discrimination algorithms.
\end{abstract}

\section{Introduction}

Accurate discrimination between SVT and VT in current ICD is to the date a widely scrutinized area of research. While the arrhythmia detection based on the ventricular heart-rate is precise enough for ventricular fibrillation episodes, there is an overlap between SVT and VT cycle-ranks leading to a number of inappropriate shocks, estimated amidst 10 and $30 \%$ [1] in unicameral devices. The addition of an atrial lead in dual chamber devices and the conceiving of SVT-VT discrimination algorithms based on the AV-sequence has improved the detection accuracy [2]. Nevertheless, AV-sequence based algorithms can still fail when facing to $\mathrm{VT}$ with $1: 1$ retrograde conduction [3], where a delayed electrical propagation will produce a series of long VA time intervals appearing as a series of short AV time intervals. A similar situation of erroneous arrhythmia classification can be found in long AV atrial tachycardias, which represent the $63 \%$ of the dual chamber ICD inappropriately delivered therapies [4].

We propose a new model, the atrial causality criterion, describing the relationship of the atrial and the ventricular ICD stored EGM when 1:1 conduction is present, together with its algorithmic implementation. We aimed to analyze the feasibility of the atrial causality as a criterion for discriminating 1:1 SVT-VT, not to propose a new ICD arrhythmia discrimination algorithm. Hence, no effort is made in order to reduce the computational burden of our method. If the criterion is shown to be accurate enough, the next step will consist on the development of simplified algorithms based on the atrial causality principle.

The paper is organized as follows. The model for the cardiac electrical activation in 1:1 AV rhythms when simultaneous atrial and ventricular EGM are available is first presented. Next, the algorithmic implementation for this model is provided. Examples for records of SR, SVT and VT are examined, and a first approach to the algorithmic stability and robustness is accomplished. Finally, conclusions and future lines of development are drawn.

\section{Atrial causality model}

Be the depolarization of the atria originated at the sinus node cells, an atrial ectopic focus or an atrial sustained conduction circuit. This phenomenon can be represented by a discrete-time impulse train where each impulse occurs at the atrial activation time, these times being $\left\{n_{k}, k \in \mathbf{Z}\right\}$, this is,

$$
x[n]=\sum_{k=-\infty}^{+\infty} \delta\left[n-n_{k}\right]
$$


where $\delta[n]$ denotes the Dirac's discrete-time sequence,

$$
\delta[n]= \begin{cases}1, & \text { if } n=0 \\ 0, & \text { otherwise }\end{cases}
$$

Let $h_{1}[n]$ represent the atrial depolarization that responses to each atrial impulse, $h_{3}[n]$ the electrical activation raising when the AV node depolarizes, and $h_{2}[n]$ a linear filter containing the delay between the atrial and the AV node activations, i.e.,

$$
h_{2}[n]=\delta\left[n-n_{o}\right]
$$

Then, the atrial and ventricular ICD stored EGM, $a[n]$ and $v[n]$, can be betoken by

$$
\begin{aligned}
& a[n]=x[n] * h_{1}[n] \\
& v[n]=x[n] * h_{2}[n] * h_{3}[n]
\end{aligned}
$$

where * denotes the discrete-time convolution between two sequences [5], and it is calculated by

$$
y[n]=x[n] * h[n]=\sum_{k=-\infty}^{+\infty} x[k] \cdot h[n-k]
$$

Equations $(1,4,5)$ are a valid convolutional model whenever the $n_{k}$ time instants remain separated enough to avoid the interference between consecutive responses. This is a commonly fulfilled condition in sustained rhythms, as far as both atrial and ventricular activation are narrower than the time interval between consecutive cycles. Additive gaussian noise is not included in the model for notation simplicity, but it could be easily inserted at the response filter outputs. All of these elements are depicted in Figure 1.

When the atrial and ventricular electrical responses, together with the atrial activation sequence, are known, the problem reduces to estimate the delay filter $h_{2}[n]$. If the sustained rhythm is in fact atrial originated, $h_{2}[n]$ is a causal system with $n_{o}>0$ and the atrial activation is causing the ventricular activation. On the contrary, if the tachycardia is bearing in the ventricular level then the ventricular arrhythmia mechanism is driving the atrial activation, the filter will be characterized by an anti-causal impulse response, and $n_{o}<0$.

\section{Algorithmic implementation}

The algorithmic implementation for the atrial causality model described above consists on three main steps.

- Impulse Response Generation. The process starts by estimating the impulse responses for the atrial and the ventricular channels. For each record, the EGM maximum amplitudes are detected with an exponentially decaying

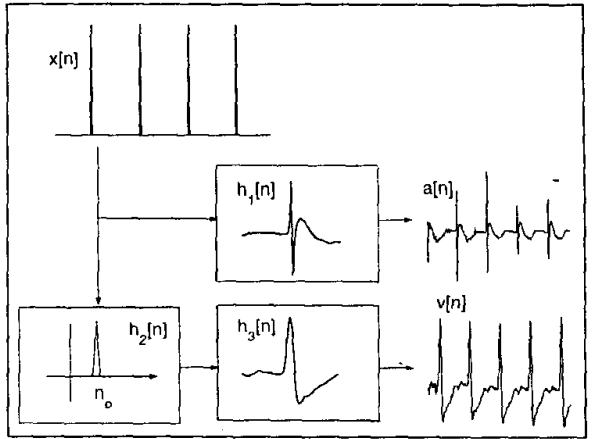

Figure 1. Scheme of the atrial causality model.

threshold. The EGM are centered with respect to this maximum and segmented on vectors of length the $90 \%$ its estimated cycle-length. The averaging of these vectors for each channel gives raise to the two templates, $\mathbf{h}_{\mathbf{1}}$ and $\mathbf{h}_{\mathbf{3}}$, characterizing $h_{1}[n]$ and $h_{3}[n]$.

- Sparse Activation Estimation. The next process is to obtain an estimate of the sparse activation in the atria. For this purpose, the following functional is minimized,

$$
\mathbf{e}_{\mathbf{a}}(\mathbf{x})=\left\|\mathbf{a}-\mathbf{H}_{1} \cdot \mathbf{x}\right\|_{1}
$$

constrained to

$$
\begin{aligned}
0 & \leq \mathbf{x} \\
u_{a} & \leq \operatorname{sparse}(\mathbf{x})
\end{aligned}
$$

where $\mathbf{x}$ is the vectorial representation of the atrial activation sequence, $\mathbf{a}$ is the vector containing the observed atrial EGM record samples, $\mathbf{H}_{1}$ is the convolution matrix obtained from $\mathbf{h}_{\mathbf{1}},\|\cdot\|$ denotes the $L_{1}$ norm of the vector, and sparse $(\cdot)$ represents the non-zero elements of a vector. Equation 7 is minimized with simple stochastic gradient descent. In order to provide a sparse solution for $\mathbf{x}$, the projection onto the convex sets defined by Equations $(8,9)$ is made at each gradient step. An appropriate value for $u_{a}$ was found in $0.15 \cdot \max (\mathbf{x})$. The solution is clipped to a unit delta train in order to remove the low-frequency amplitude oscillations in the atrial EGM, giving a solution vector $\bar{x}$.

- Delay Filter Estimation. A vector $w$ is built from $\bar{x}$ and $\mathbf{h}_{\mathbf{3}}$ in order to characterize $x[n] * h_{3}[n]$. The filter length $L$ is set to an odd number for providing the same extent both the causal and the anti-causal filter counterparts. The minimized functional in this case is

$$
\mathbf{e}_{\mathbf{v}}\left(\mathbf{h}_{2}\right)=\left\|v-W \cdot h_{2}\right\|_{1}
$$

constrained to:

$$
\begin{aligned}
\mathbf{0} & \leq \mathbf{h}_{\mathbf{2}} \\
u_{v} & \leq \operatorname{sparse}(\mathbf{x})
\end{aligned}
$$




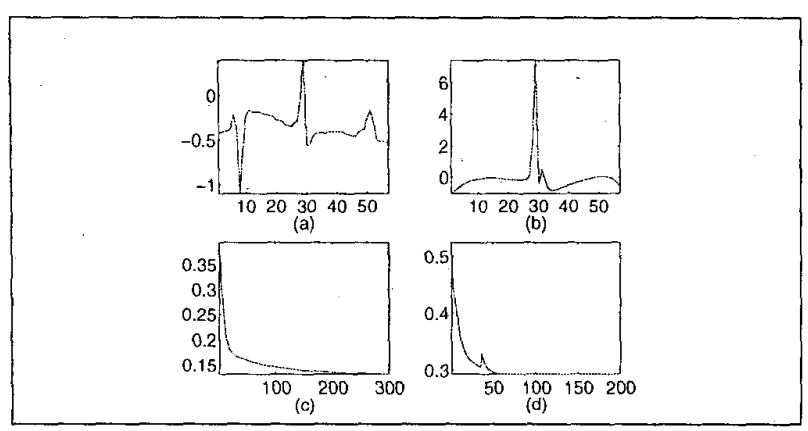

Figure 2. Optimization procedure for $1: 1 \mathrm{VT}$ episode. (a) Template for the atrial impulse response. (b) Template for the ventricular impulse response. (c) The $L_{1}$ error for the sparse activation estimation process. (d) Idem for the delay filter estimation process

where $\mathbf{v}$ contains the ventricular channel samples, and $\mathbf{W}$ is the convolution matrix obtained from $w$. This functional is again optimized with stochastic gradient descent and constrain projection. In this case, a value of $u_{v}=$ $0.45 \cdot \max \left(\mathbf{h}_{2}\right)$ was found adequate for the filter estimation process.

The number of gradient descent steps was set to $\mathrm{N}=300$ and $\mathrm{N}=200$ for the sparse activation and the delay filter estimations, respectively.

\section{Examples: SR, SVT and VT records}

To test whether the atrial causality model is a reasonable approximation, we examined several tachycardia episodes from ICD stored EGM, from a third generation implanted ICD (GEM-DR 7271, Medtronic, Minneapolis, MN, USA). The atrial and the ventricular EGM sources were ATIP/ARING (bipolar EGM in the atria) and HVA/HVB (between the subpectoral can and the defibrillation coil in the left ventricle). Both simultaneous channels were digitized $(128 \mathrm{~Hz}, 8$ bits, range $\pm 7.5 \mathrm{mV})$ during $1: 1$ sustained SVT, VT and basal SR. All the EGM were obtained from spontaneous episodes. The EGM analysis and the arrhythmia classification was performed by one of the authors (F.A.) with expertise in the field.

Three records were selected: SR, 1:1 SVT and 1:1 VT. The atrial causality model was verified in all of them, which is shown in Figures $(2,3)$. For the three rhythms, the activation sequence was found to be properly estimated by a sparse series, and the coherence is verified by the reduced error between the atrial real and the atrial approximated EGM. The adequacy of the estimated delay filter is verified in the same way, by contrasting the real and the approximated ventricular EGM.

Lower panel of each sub-figure in Figure 3 depicts the estimated delay filter, for a length of $L=101$ samples $(\simeq$ $800 \mathrm{~ms}$ ) in all the cases. In the atrial-generated rhythms (SR

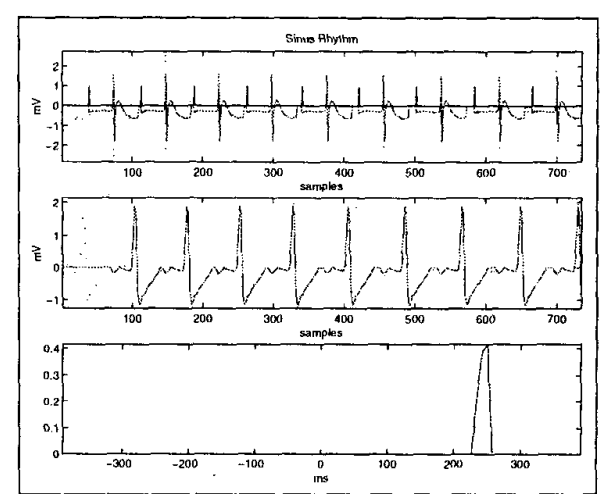

(a)

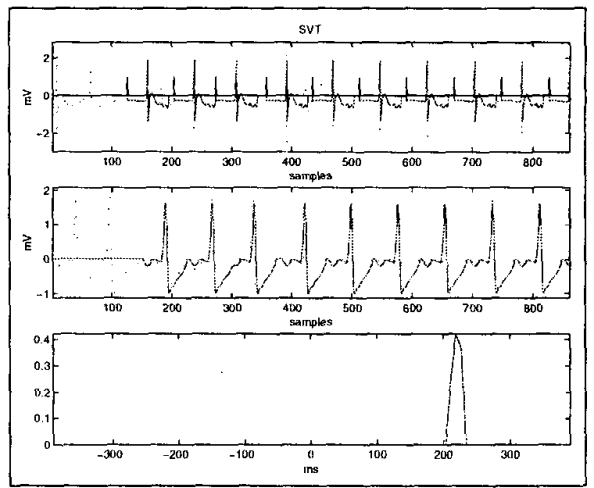

(b)

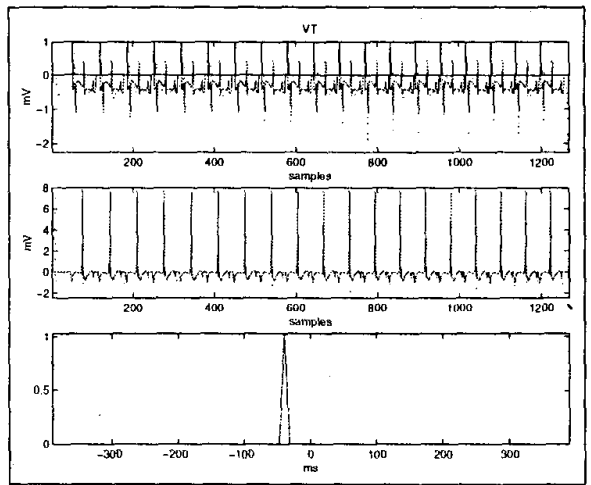

(c)

Figure 3. Atrial causality model for (a) SR (b) SVT (c) VT. Each panel depicts- Up: recorded (dotted) and estimated (solid) atrial EGM, and sparse activation estimation-Middle: recorded (dotted) and estimated (solid) ventricular EGM-Down: estimated delay filter.

and SVT) the delay is $n_{0}>0$, the filter is causal and so it is the relationship between both channels. On the opposite, the VT filter is obtained an anti-causal shape, with $n_{o}<0$. Note the filter length being several times the tachycardia cycle in both SVT and VT.

Another interesting issue is to observe this VT case 


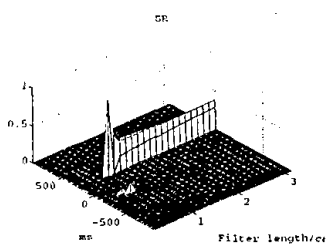

(a)

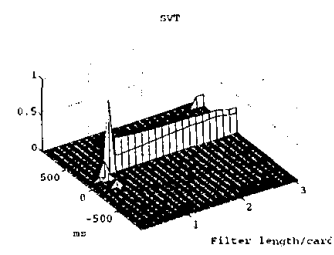

(b)

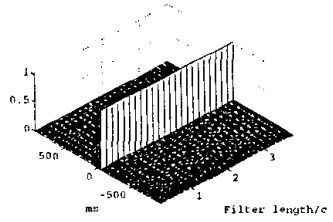

(c)

Figure 4. Variation of the delay filter shape estimation with the filter length. (a) SR, (b) SVT, (c) VT. The filter length is normalized with respect to the ventricular cycle length of the record.

exhibiting far-field sensing in the atrial lead from the ventricular activity (see atrial template in Figure 2-a). This is not a problem for the method, and it has also been tested (not included here) the far-field sensing does not either deteriorate the SVT detection. Another tested characteristic is the algorithm also works in both dissociated and $\mathrm{n}: 1$ or $1: n(n>1)$ arrhythmia, but this is not an issue for the current dual-chamber ICD algorithms, and hence it does not suppose a preferential future research line.

Finally, the robustness of the algorithms is briefly tested. Apart from the thresholds and the number of error minimization steps, an important parameter from a model point of view is the filter length. If the filter is given a long enough shape, possible solutions include not only the "nearest" AV time interval, but also the nearest VA time interval and several more, allowing to give any solution of them. If the filter keeps giving the same solution despite of a large length, it will be taken into account the small variations of the source and hence approximating the correct delay filter impulse response. However, if it takes the nearest solution, or becomes multi-modal, the method will not be useful for 1:1 SVT-VT discrimination.

For the SR, SVT and VT records, the delay filter estimation process was made by changing the parameter $L \in(11,241)$ samples. The obtained filters in each rhythm were centered and padded with zeros, in order to have a visual representation of the estimation. Figure 4 shows these variations. Whenever the filter length is too short for building a proper solution, it is not working. From the moment it reaches the proper $\mathrm{AV}$ (or VA) time interval it stabilizes for several cycle lengths. The goodness is not indefinite, as for the SVT example it becomes multi-modal for a length greater than 3 cycles. Nevertheless, the working zone can be considered wide enough for a proper model fitting.

\section{Conclusions}

The atrial causality model appears to be an appropriate and robust criterion to discriminate 1:1 SVT-VT. This principle is independent of the EGM lead configuration, the presence of far-field sensing and the delay filter length. Nevertheless, some issues remain to be explored in order to derive ICD implementable algorithms. It would be desirable to test the criterion validity in a wider database of $1: 1$ records. A measure of the robustness of the algorithm should also be obtained from the comparison with other deconvolution algorithms. Simplified, yet adequate rules could eventually be derived if the principle is shown to be effective.

\section{References}

[1] Barold H, Newby K, Tomassoni G, Kearney M, Brandon J, Natale A. Prospective Evaluation of New and Old Criteria to Discriminate Between Supraventricular and Ventricular Tachycardia in Implantable Defibrillators. PACE July 1998; 21:1347-1355.

[2] Swerdlow C, Schels W, Dijkman B. Detection of atrial fibrillation and flutter by a dual-chamber implantable cardioverter-defibrillator. Circulation 2000;101:878-85.

[3] Külkamp V, Dörnberger V, Mewis C. Clinical experience with the new detection algorithms for atrial fibrillation of a defibrillator with dual chamber sensing and pacing. J Cardiovasc Electrophysiol 1999;10.

[4] Dijkman B, Wellens H. Dual chamber arrhythmia detection in the implantable cardioverter defibrillator. J Cardiovasc Electrophysiol oct 2000;11:1105-15.

[5] Oppenheim A, Willsky A, Young I. Signals and Systems. Englewood Cliffs, New Jersey: Prentice Hall, 1983.

Address for correspondence:

José Luis Rojo Álvarez

Sur 318, Escuela Politécnica, Universidad de Alcalá

Alcalá de Henares, Madrid. 28007 Spain.

joseluis.rojo@uah.es 